\title{
Healthcare Professionals' Perceptions of Adoption of EHRs in Primary Care in Saudi Arabia
}

\author{
Bander Dowahi ALANAZI ${ }^{\mathrm{a}, 1}$ and Bella NAPTSOK ${ }^{\mathrm{b}}$ \\ ${ }^{a}$ College of Health and Medicine, University of Tasmania, Launceston, Australia \\ ${ }^{\mathrm{b}}$ Department of Literature, Adyghe State University, Maykop City, Russia
}

\begin{abstract}
EHRs are crucial for the provision of high-quality healthcare. However, their adoption and utilization are influenced by several factors, including users' perceptions. This study evaluated the perceptions of Saudi healthcare professionals towards the adoption of EHRs in primary care. All healthcare professionals working in primary care centers in Riyadh city were surveyed, resulting in a $65.9 \%$ response rate. Overall, the respondents had a positive perception of EHRs, as demonstrated by high agreement levels across all benefits and low with obstacles. There was also a positive relationship between perceived benefits and satisfaction with EHRs but negative with obstacles to adoption.
\end{abstract}

Keywords. electronic health records, healthcare professionals, perceptions

\section{Introduction}

Electronic health records (EHRs) provide several benefits that improve the quality of health care service provision [1]. These may include improving communication between healthcare providers and patients, clinical data documentation, and access to patient records [2, 3]. However, concerns with the system's limitations, including but not limited to increased risk of medical errors, complexity in use, and increased workload, also persist [4]. Users' perceptions of these factors can influence the acceptance, adoption, and use of EHRs $[5,6]$. This study evaluates perceptions of healthcare professionals towards the adoption of EHRs in primary care in order to inform adoption in such settings.

\section{Methods}

All $(1,710)$ healthcare professionals working in primary care centers in Riyadh city, Saudi Arabia, were surveyed to explore their perceptions regarding benefits and obstacles to adopting EHRs, as well as satisfaction with the systems. A 33-item survey tool adopted from a Turkish study [7] and validated for the Saudi context [8] was deployed online using REDCap to collect data between 11/30/2017 and 01/30/2018. Prior consent was obtained, and all the responses were anonymous.

\footnotetext{
${ }^{1}$ Corresponding Author, Bander Dowahi Alanazi, College of Health Science, University of Tasmania, Launceston, Australia; E-mail: bander12@hotmail.com.
} 


\section{Results}

A total of 1127 participants $(65.9 \%)$ completed the survey. The perceptions varied across the items for benefits, obstacles, and satisfaction with EHRs in primary care. The agreement with benefit statements ranged between $63.5 \%$ for an EHR 'reduces medical errors' and $77.1 \%$ for an EHR 'decreases paper-based documentation' [8]. Similarly, the agreement levels for satisfaction items were also high (65.8\% to 78.8\%). Conversely, the obstacle statements had low agreement levels except for an EHR system 'needs frequent revisions due to technological developments', with a $45.3 \%$ agreement. In addition, there was a strong positive association with perceived benefits (canonical correlation coefficient, $r=0.91)$ and medium association with perceived obstacles $(r=0.45)$.

\section{Discussion}

The results show that primary healthcare professionals in Saudi Arabia generally have a positive perception of EHRs, which is consistent with previous studies [9, 10]. The positive perceptions could be related to the benefits of EHRs, as demonstrated by the results of a positive association between perceptions of benefits and satisfaction with EHRs. These findings suggest that primary care professionals in Saudi Arabia are more likely to adopt EHRs mainly due to their perceived usefulness as opposed to perceived obstacles. However, technological challenges could remain a common barrier to adopting EHRs and must be adequately addressed to improve perception and adoption.

\section{References}

[1] Manca DP. Do electronic medical records improve quality of care?: Yes. Canadian Family Physician. 2015;61(10):846-7.

[2] Hoover R. Benefits of using an electronic health record. Nursing2018. 2016;46(7):21-2.

[3] Schopf TR, Nedrebø B, Hufthammer KO, Daphu IK, Lærum H. How well is the electronic health record supporting the clinical tasks of hospital physicians? A survey of physicians at three Norwegian hospitals. BMC health services research. 2019;19(1):1-9.

[4] Menachemi N, Collum TH. Benefits and drawbacks of electronic health record systems. Risk management and healthcare policy. 2011;4:47.

[5] Kruse CS, Kristof C, Jones B, Mitchell E, Martinez A. Barriers to electronic health record adoption: a systematic literature review. Journal of medical systems. 2016;40(12):252.

[6] Garavand A, Mohseni M, Asadi H, Etemadi M, Moradi-Joo M, Moosavi A. Factors influencing the adoption of health information technologies: a systematic review. Electronic physician. 2016;8(8):27138. Epub 2016/10/21. doi: 10.19082/2713. PubMed PMID: 27757179; PubMed Central PMCID: PMCPmc5053450.

[7] Secginli S, Erdogan S, Monsen KA. Attitudes of health professionals towards electronic health records in primary health care settings: a questionnaire survey. Informatics for Health and Social Care. 2014;39(1):15-32.

[8] Alanazi B. Evaluating the healthcare professionals' perceptions about the adoption of electronic health records in primary care centres in Riyadh City, Saudi Arabia: University of Tasmania; 2020.

[9] Al-Harbi A. Healthcare providers' perceptions towards health information applications at King AbdulAziz Medical City, Saudi Arabia. International Journal of Advanced Computer Science and Applications. 2011;2(10):10-3.

[10] Shaker HA, Farooq MU, Dhafar KO. Physicians' perception about electronic medical record system in Makkah Region, Saudi Arabia. Avicenna journal of medicine. 2015;5(1):1-5. Epub 2015/01/28. doi: 10.4103/2231-0770.148499. PubMed PMID: 25625082; PubMed Central PMCID: PMCPmc4296390. 